Northern Ireland Neurology Service, Belfast, $N$ Ireland GV McDonnell JA Lyttle

\section{Department of Ophthalmology, Royal Victoria Hospital, Belfast, $N$ Ireland \\ G Silvestri \\ Regional Virus Laboratory, Royal Victoria Hospital, Belfast, N Ireland PV Coyle \\ Correspondence to Dr GV McDonnell Department of Neurology, Royal Victoria Hospital, Grosvenor Road, Belfast BT12 6BA, N Ireland}

Accepted 28 February 1996

\title{
Acute visual disturbance in a young adult
}

\author{
GV McDonnell, G Silvestri, JA Lyttle, PV Coyle
}

A 27-year-old, right-handed, professional diver presented with a recent onset of periocular pain and blurring of vision in the left eye. Visual acuity was $6 / 36$ in the left eye and 6/6 in the right eye. Examination of the anterior segment showed evidence of keratic precipitates and of an intense anterior chamber reaction. Dilatation of the pupil with cyclopentolate $1 \%$ and phenylepherine $10 \%$ revealed the appearance demonstrated in the figure. There appeared to be no involvement of the optic nerve.

Three months earlier the patient was discovered wandering confusedly on the deck of his dive barge an hour or two after making a routine dive to a depth of 30 metres. For the previous 72 hours he had been off work because of an upper respiratory tract infection and headache.

On examination the patient was drowsy and pyrexic. There was generalised hyper-reflexia with non-sustained clonus on the left side. The left plantar response was extensor. Kernig's sign was negative. Pupillary responses were sluggish but equal and fundoscopy revealed early blurring of both optic discs. Brain computed tomography (CT) demonstrated an ill-defined hypodensity in the right temporal region with effacement of the right lateral ventricle. Magnetic resonance imaging (MRI) showed hypodensity in both the right temporal and parietal lobes that enhanced with intravenous contrast and produced mass effect on the right lateral ventricle. Electroencephalography (EEG) showed marked generalised slowness, particularly in the right hemisphere, with focal secondarily generalised epileptic discharges.

Following treatment, the patient's condition improved with resolution of lateralising neurological signs. There was, however, profound amnesia for recent events and a repeat MRI scan of brain four weeks later displayed atrophy of both temporal lobes.

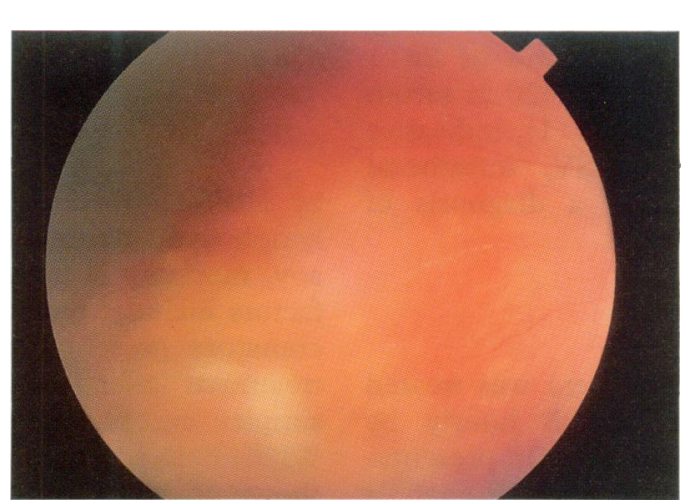

Figure Fundal photograph of left eye

\section{Questions}

1 What does the fundal photograph show?

2 What is the differential diagnosis?

3 What was the reason for the patient's initial presentation and what further investigation would have been useful diagnostically? 


\section{Answers}

QUESTION 1

The figure shows a focus of retinal necrosis in the 8 o'clock position, together with an associated vasculitis and an overlying vitritis. Taken alongside the anterior chamber appearance, the features are those of acute retinal necrosis (box 1).

\section{QUESTION 2}

Several viruses (box 2) have been implicated in the aetiology of acute retinal necrosis. Toxoplasma may also cause a retinitis with significant vitreous reaction as, more rarely, may Candida albicans, Epstein-Barr virus, and cryptococcus neoformans.

\section{QUESTION 3}

The initial presentation with a prodromal illness of headache and malaise followed by altered consciousness, pyrexia and the development of focal neurological signs is typical of viral encephalitis. Neuroimaging revealing low attenuation in the temporal lobes and generalised slowing and periodic lateralised epileptiform discharges on EEG was also supportive diagnostically. Although cerebrospinal fluid (CSF) analysis was not performed initially because of concern about raised intracranial pressure, CSF examined five weeks after onset of illness revealed a protein of $1.04 \mathrm{~g} / \mathrm{l}$ and no inflammatory cells. Herpes simplex virus (HSV) antibody titres were $1: 10240$ in serum and $1: 512$ in CSF giving a ratio of $20: 1$ (measles antibody titres were $1: 320$ in serum and $1: 2$ in CSF, a ratio of $160: 1$ ), indicative of recent infection with $\mathrm{HSV}$, intrathecal production of antibody and a diagnosis of herpes simplex encephalitis.

\section{Treatment}

For the initial illness, the patient was treated with $10 \mathrm{mg} / \mathrm{kg}$ of acyclovir, 8 hourly by intravenous infusion for 10 days. On presentation with acute retinal necrosis, CSF analysis revealed an elevated protein of $0.85 \mathrm{~g} / 1$ and 31 leucocytes $/ \mu \mathrm{l}$. Serum and CSF HSV antibody titres were $1: 10240$ and $1: 2048$, respectively, a ratio of $5: 1$ (measles serum:CSF ratio remained at $160: 1)$. He received a similar course of intravenous acyclovir followed by $400 \mathrm{mg}$ orally five times daily for one week and then $200 \mathrm{mg}$ five times daily for one week. This resulted in clearing of the cellular reaction and improvement in visual acuity to $6 / 9$. There was no evidence of peripheral retinal breaks or retinal detachment. Serum and CSF antibody titres had fallen to $1: 3200$ and and $1: 128$, respectively, and nested polymerase chain reaction analysis of the CSF, using primers targeted to the glycoprotein D gene of HSV, was negative.

\section{Discussion}

Herpes simplex encephalitis has an annual incidence of one in 250000-300 000. In adults and older children it is usually caused

\begin{tabular}{|l|}
\hline Features of acute retinal necrosis \\
\hline - panuveitis \\
- vitritis \\
- retinal arteritis \\
- frequent recrol negmatogenous retinal detachment \\
\hline
\end{tabular}

Box 1

Acute retinal necrosis: aetiology

- varicella-zoster virus

- herpes simplex virus types $1 \& 2$

- cytomegalovirus

Box 2

Herpes simplex encephalitis:
learning points
- intravenous acyclovir is the treatment of
choice
- standard 7 - 10 day regimes of acyclovir may be
insufficient to prevent relapse
- acute retinal necrosis is a recognised
complication and may occur several months
after the initial infection
- polymerase chain reaction may provide the
means of assessing optimal duration of antiviral
therapy

Box 3

by HSV-1, whereas HSV-2 causes a benign lymphocytic meningitis. Without specific therapy, mortality is up to $70 \%$. Poor prognostic factors include: patients over 30 years of age, comatose presentation and establishment of treatment after widespread bilateral herpes simplex encephalitis proven by CT or MRI. Whilst both acyclovir and vidarabine have been shown to be efficacious, acyclovir is the less toxic and more effective, reducing six-month mortality to $19 \%$. $^{1}$

This patient did indeed make a good initial recovery following treatment with a standard regime, the severe residual cognitive impairment being unsurprising given the degree of bilateral temporal lobe atrophy. His subsequent relapse has been similarly described in both children and adults despite acyclovir therapy. This has been attributed to a comparatively short duration of therapy allowing either regrowth or reactivation of residual viruses. Whilst the occurrence of herpes simplex encephalitis along with acute retinal necrosis is also well recognised, the delay of three months between them is more unusual, although herpes simplex retinitis has been documented as occurring one month ${ }^{2}$ and even two years after herpes simplex encephalitis. $^{3}$

Acute retinal necrosis itself is an uncommon ocular disorder, first described in 1971 (box $1)^{2}$ It is unilateral in $65 \%$ of cases, can occur at 
all ages and affects both sexes equally. The effect on vision can be devastating due to retinal arteritis, retinal necrosis and rhegmatogenous retinal detachment. It is most commonly caused by reactivation of latent infection by varicella zoster virus or HSV but has also been described occurring during herpes simplex encephalitis, soon after shingles and within a month of the onset of chickenpox. Most patients are healthy with no significant medical history although immunosuppressed patients following renal transplantation and acquired immune deficiency syndrome patients have been affected, ${ }^{4}$ making HIV testing relevant when risk factors are identified by history or examination.

A definitive approach to management of acute retinal necrosis has yet to be established. The regime in this relatively mild case proved adequate but in more complicated or less responsive cases, treatment with systemic steroids and a more prolonged course of acyclovir may be appropriate. In any event,

1 Whitley RJ, Alford CA, Hirsch MS, et al. Vidarabine versus acyclovir therapy in herpes simplex encephalitis. $N$ Engl $\mathcal{F}$ Med 1986; 314: 144-9.

2 Gartry DS, Spalton DJ, Tilzey A, Hykin PG. Acute retinal necrosis syndrome. $B r f$ Ophthalmol 1991; 75: 292-7.

3 Sekizawa T, Hara S, Kiyosawa M, Openshaw H, Kogure K. Acute retinitis 2 years after recovery from herpes simplex encephalitis. Neurology 1991; 41: 456. this and other reports of relapse following herpes simplex encephalitis raise doubts about the adequacy of the conventional 10-day regime of acyclovir for eradication of HSV. The recent availability of polymerase chain reaction for detection of HSV DNA has resulted in the recommendation that it should now be the standard for the diagnosis of herpes simplex encephalitis; it also provides the prospect of establishing more accurately the appropriate duration and adequacy of anti-viral therapy in each individual case. A controlled trial to determine if prolonged acyclovir therapy based on the presence of HSV DNA in the CSF can prevent relapse is underway. ${ }^{5}$

\section{Final diagnosis}

Acute retinal necrosis occurring as a late complication of herpes simplex encephalitis.

Keywords: retinal necrosis, herpes simplex encephalitis, acyclovir, polymerase chain reaction

4 Friedman SM, Mames RN, Sleasman JW, Whitcup SM Acute retinal necrosis after chickenpox in a patient with acquired immune deficiency syndrome. Arch Ophthalmol 1993; 111: $1607-8$

5 Kaneko K, Watanabe H, Hikita M, Tokita A, Yabuta K Cessation of antiviral therapy for herpes simplex encephalitis. F Pediatr 1994; 124: 490

\title{
An unusual cause of large bowel obstruction
}

\author{
Faisal Mihaimeed, Audrey Yong, Trevor Paes
}

A 42-year-old woman was re-admitted 10 days following total abdominal hysterectomy for fibroids with abdominal pain and vomiting. She had undergone Nissen's fundoplication for hiatus hernia via a left thoracotomy six months previously. An initial diagnosis of large bowel obstruction was made following routine investigations, which revealed distended loops of small and large bowel with normal chest X-ray. She was initially managed with intravenous fluids and nasogastric suction. Fifteen hours later she developed severe left-sided chest pain with shortness of breath. A repeat chest X-ray was performed (figure).

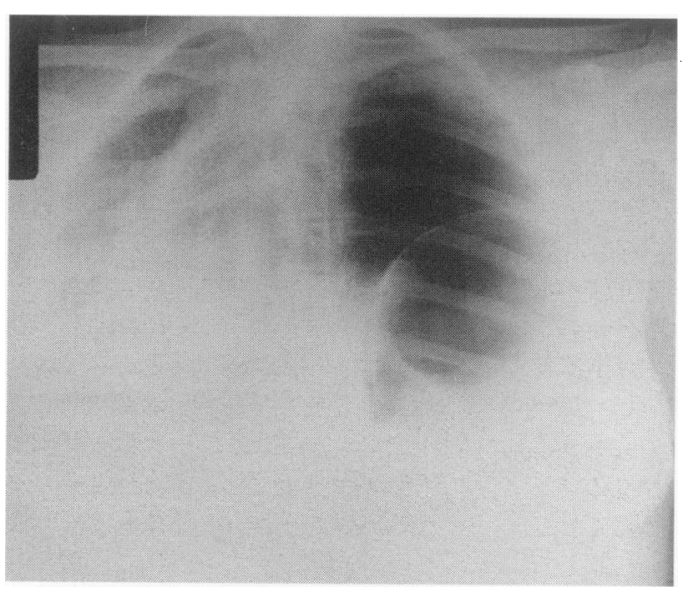

Figure Chest X-ray

\section{Here Chest X-ray}

\section{Questions}

1 What is the diagnosis?

2 Describe the immediate management of this patient
Department of

General Surgery, The

Hillingdon Hospital

Trust,

Pield Heath Road,

Uxbridge UB8 3NN,

UK

F Mihaimeed

A Yong

T Paes

Correspondence to $\mathrm{Dr} A$ Yong

Accepted 7 March 1996 